\title{
Reduced expression of COMT in placenta correlates with term preeclampsia
}

\author{
Baoling Lai, Chenhong Wang", Lu Zhou, Jie Wang, Huifen Wu \\ Shenzhen Maternity and Child Healthcare Hospital, South Medical University, Shenzhen, China \\ Email: *pauling925@126.com
}

Received January $19^{\text {th }}, 2013$; revised March $17^{\text {th }}, 2013$; accepted April 15 ${ }^{\text {th }}, 2013$

Copyright (C) 2013 Baoling Lai et al. This is an open access article distributed under the Creative Commons Attribution License, which permits unrestricted use, distribution, and reproduction in any medium, provided the original work is properly cited.

\begin{abstract}
Objective: To investigate the expression of catechol-Omethyltransferase (COMT) in placentas from term preeclampsia patients in a Chinese cohort and to analyze the relationships between COMT and mean artery pressure (MAP), serum uric acid and 24-hour urine protein. Methods: 15 term pregnant patients with preeclampsia and 15 normal pregnant women were enrolled. Clinical characteristics and placental tissue were collected. Placental COMT expression was determined by quantitative real-time PCR technique and immunohistochemistry. Results: The level of COMT mRNA in placentas from the preeclampsia group was significantly decreased than that in the normal pregnant women $(0.00179 \pm 0.00051$ vs. $0.00289 \pm 0.00066$, $t=5.145, P<0.001)$. Adjusted by age and body mass index (BMI), the COMT expression was negatively correlated with serum uric acid $(r=-0.660, P<$ $0.001)$, 24-hour urine protein $(r=-0.717, P<0.001)$ and MAP $(r=-0.619, P<0.001)$. Conclusion: The decreased expression of COMT in placentas from term preeclamptic patients and the significant relation between COMT and severity index of preeclampsia suggest that COMT may play a role in the pathogenesis of term preeclampsia.
\end{abstract}

Keywords: Catechol-O-Methyltransferase; Preeclampsia; Placenta

\section{INTRODUCTION}

Although preeclampsia is associated with increased maternal and perinatal morbidity and mortality worldwide, our understanding of its pathophysiology and etiology is still incomplete. But it is beyond doubt that the placenta contributes to the pathogenesis of preeclampsia because its development requires the presence of the placenta and

"Corresponding author. delivery of the placenta is the most effective way to treat preeclampsia.

Last 6 years some research findings support the hypothesis that the symptoms are related to a decrease in free vascular endothelial growth factor (VEGF) in the maternal blood, as a result of capture by sFlt1, the soluble form of VEGF-receptor1 [1]. However, not all women with preeclampsia have increased sFlt1 and endoglin concentrations. And further upstream, the mechanisms at work in the primary placental defect are unknown. Recently, an ex vivo study using genetic COMT knockout mice suggested that placental COMT deficiency induced preeclampsia due to an absence of 2-methoxyestradiol (2-ME), a product of COMT. Administration of 2-ME to COMT-deficient mice reverts the PE-like phenotype without toxicity [2]. Moreover, 2-ME has been shown to facilitate the development of the placenta and embryo by promoting the establishment of the uteroplacental circulation under hypotic conditions [3]. Together, these results suggest that COMT and 2-ME deficiency might play a significant role in the development of preeclampsia. Although these studies provide an attractive hypothesis implicating reduced COMT expression in the pathogenesis of preeclampsia, clinical evidence confirming that reduced COMT expression occurs in preeclampsia has been limited. Furthermore, placentas obtained from pregnancies complicated by term preeclampsia have not been investigated. The early-onset preeclampsia may be responsible for the largest burden of perinatal and maternal morbidity and mortality. But in some cases, late-onset preeclampsia is also lifethrea-tening to the mother and fetus [4]. Therefore, we decided to conduct a case-control study to investigate placental COMT expression in patients with term preeclampsia in a Chinese cohort.

\section{MATERIALS AND METHODS}

\subsection{Patients and Sample Collection}

Samples were collected from term pregnant women re- 
cruited from the department of obstetrics at Shenzhen Maternity and Child Healthcare Hospital between April 2012 and August 2012. The local ethical committee approved this study and written informed consent was obtained from all the patients. Placental tissue was obtained during the deliveries by cesarean section from normal term pregnancies $(n=15)$ and term pregnancies complicated by preeclampsia $(n=15)$. Preeclampsia was defined as a pregnancy-specific syndrome that occurred after 20 weeks of gestation and was characterized by systolic pressure of $140 \mathrm{mmHg}$ or higher or diastolic pressure of $90 \mathrm{mmHg}$ or higher on two occasions longer than 6 hours apart, occurring with proteinuria $(>300 \mathrm{mg} /$ day).

Placental tissue was collected immediately after delivery by caesarean section. Tissue was obtained from the maternal site of the placenta, about $3 \mathrm{~cm}$ from the area of the cord insertion. Specimens were cut into small pieces and washed with ice-cold PBS. Samples for collected for RNA extraction were immersed in RNAlater (Ambion) overnight at $4^{\circ} \mathrm{C}$ and transferred to $-20^{\circ} \mathrm{C}$ for long-term storage. Samples for immunohistochemistry were fixed in $4 \%$ formalin.

\subsection{Real-Time PCR}

Total RNA from placental samples $(50 \mathrm{mg})$ was obtained using $1 \mathrm{ml}$ of Trizol (Invitrogen) according to the manufacturer's instructions. At the end of the procedure, RNA pellets were briefly air-dried for $10 \mathrm{~min}$, dissolved in DEPC water. RNA purity and concentration were verified using a Nanodrop spectrophotomer (Thermo Scientific); all RNA samples had an $\mathrm{A}_{260} / \mathrm{A}_{280}$ ratio of 1.80 - 2.0. Total RNA was reverse-transcribed to cDNA in a $20 \mu \mathrm{l}$ reaction using oligo-dT primers according to manufacturer's protocol (MBI). cDNA was diluted with nuclease-free water (Thermo Scientific). Real-time PCR analysis was then performed in a fluorescent temperature cycler (SYBR Green dye (TaKaRa)) to examine the mRNA level of COMT in placental tissue with LightCycler 480 (Roche) according to the manufacturer's instructions. The PCR cycling conditions included an initial denaturation at $95^{\circ} \mathrm{C}$ for 10 seconds, followed by 50 cycles at $95^{\circ} \mathrm{C}$ for 5 seconds, $55^{\circ} \mathrm{C}$ for 8 seconds, and $72^{\circ} \mathrm{C}$ for 12 seconds. The sequences of the PCR primer pairs for COMT (84 bp) and GAPDH (110 bp) were fellows. For COMT, forward primer was

5'-TCCTTCTCAACTGCCATTCC-3', reverse primer was 5'-CCTTAGCGTTAGCGTCCG TC-3'. For GAPDH, forward primer was

5'-CAAGAAGGTGGTGAAGCAGG-3', reverse primer was 5'-AGGTGGAGGAGTGGGTGTCG-3' (Invitrogen). The cycle threshold values and COMT relative values were obtained using the software provided by the manufacturer.

\subsection{Immunohistochemistry}

The placentas were fixed in 4\% formalin for 12 to 24 hours, embedded in paraffin and cut into $4-\mu \mathrm{m}$ sections. The sections were first deparaffinised by immersion in xylene followed by rehydration by passing through descending concentrations of alcohol (100\% - 70\%) and finally immersion in running tap water. Antigen retrieval was performed by microwave heating these sections for $10 \mathrm{~min}$ in trisodium citrate buffer $(10 \mathrm{mM})$ sodium citrate, $\mathrm{PH}$ 6.0). Sections were then washed with PBS. Slides were then incubated for $2 \mathrm{~h}$ at room temperature with Rabbit anti human COMT polyclonal antibody (1:40, Abcam). After three times washing sections were incubated with MaxVision solution (Maixin Bio, China) for $30 \mathrm{~min}$. The staining was detected with DAB chromogen after $5 \mathrm{~min}$. The nuclei were counterstained by incubation with hematoxylin for $2 \mathrm{~min}$. The sections were mounted, examined and photographed. Specificity of staining was confirmed using positive control tissue. Negative controls were also performed in each staining run using no primary antibody.

\subsection{Immunohistochemical Semiquantification}

15 images from each group were taken with the microscope settings unaltered. Semiquantitative analysis of COMT expression based on the combination of staining intensity of immuno-histochemical images with the percentage of positive cells was performed by a published method [5]. Briefly, no staining is scored as $0,1 \%-10 \%$ of positive cells stained scored as $1,11 \%-50 \%$ as 2 , $51 \%-80 \%$ as 3 , and $81 \%-100 \%$ as 4 . Staining intensity is rated on a scale of $0-3$, with $0=$ negative; $1=$ weak; $2=$ moderate, and $3=$ strong. The raw data were converted by multiplying the quantity and staining intensity scores. Negative controls were stained without primary antibody.

\subsection{Statistical Analysis}

Statistical analyses were performed with SPSS software version 13.0. Clinical characteristics and placental COMT mRNA data were presented as mean \pm standard deviation (SD). The statistical significance of the results was assessed by $t$ test. The associations between COMT mRNA and clinical parameters were analyzed by partial correlation. The data from the semiquantification of immunohistoche-mical staining was analysed using a MannWhitney Test. All probability values were 2-sided and differences were considered statistically significant when $P<0.05$. 


\section{RESULTS}

\subsection{The General Characteristics of Patients}

The characteristics of patients are summarised in Table 1. The MAP, BMI and serum uric acid level were significantly higher in pregnant women with preeclampsia $(P<$ $0.05)$. There were no significant difference in maternal age, gestational age and birth weight of infants between normal pregnancies and pregnancies with preeclampsia $(P>0.05)$.

\subsection{Placental COMT-mRNA Level and Its Correlation with Clinical Parameters}

COMT-mRNA of all placentas was detected. The mRNA level of COMT in preeclamptic placentas was significantly decreased compared to that in gestation-matched normal placentas $(P<0.001$, Figure 1). When control the BMI, the level of COMT-mRNA in preeclamptic group was negatively correlated with MAP, serum uric acid and 24-hour urine protein (all $P<0.001$, Table 2).

\subsection{Expression and Immunolocalization of COMT in Placentas}

The cells that produce COMT in the placenta were identified with immunohistochemistry. COMT was primarily detected in the syncytiotrophoblasts of the placentas from preeclamptic women. A similar but more intense staining pattern was observed in the same cell types of the placentas from the normal pregnant women (Figure 2). Semiquantitative analysis indicated this reduced expression of COMT in preeclamptic placentas was significant $(P<0.05)$ (Figure 3).

Table 1. The general characteristics of patients.

\begin{tabular}{|c|c|c|c|c|}
\hline & Preeclampsia $(\mathrm{n}=15)$ & Normal $(\mathrm{n}=15)$ & $t$ & $P$ \\
\hline Maternal age (years) & $27.93 \pm 4.48$ & $29.07 \pm 4.11$ & 0.722 & 0.476 \\
\hline Delivery age (weeks) & $38.45 \pm 1.00$ & $38.86 \pm 0.64$ & 1.360 & 0.185 \\
\hline BMI $\left(\mathrm{kg} / \mathrm{m}^{2}\right)$ & $27.16 \pm 2.35$ & $25.32 \pm 2.22$ & -2.076 & $0.048^{*}$ \\
\hline MAP (mmHg) & $118.84 \pm 6.24$ & $85.84 \pm 7.09$ & -13.536 & $0.000^{* *}$ \\
\hline Uric acid $(\mu \mathrm{mol} / \mathrm{L})$ & $444.80 \pm 70.81$ & $296.87 \pm 55.83$ & -6.354 & $0.000^{* *}$ \\
\hline Birth weight (g) & $3058.33 \pm 518.67$ & $3336.67 \pm 474.52$ & 1.454 & 0.159 \\
\hline
\end{tabular}

Abbreviation: BMI, body mass index; MAP, mean average pressure. Values were expressed as mean \pm SD. ${ }^{*} P<0.05$; ${ }^{* *} P<0.001$.

placental COME relative mRNA levels

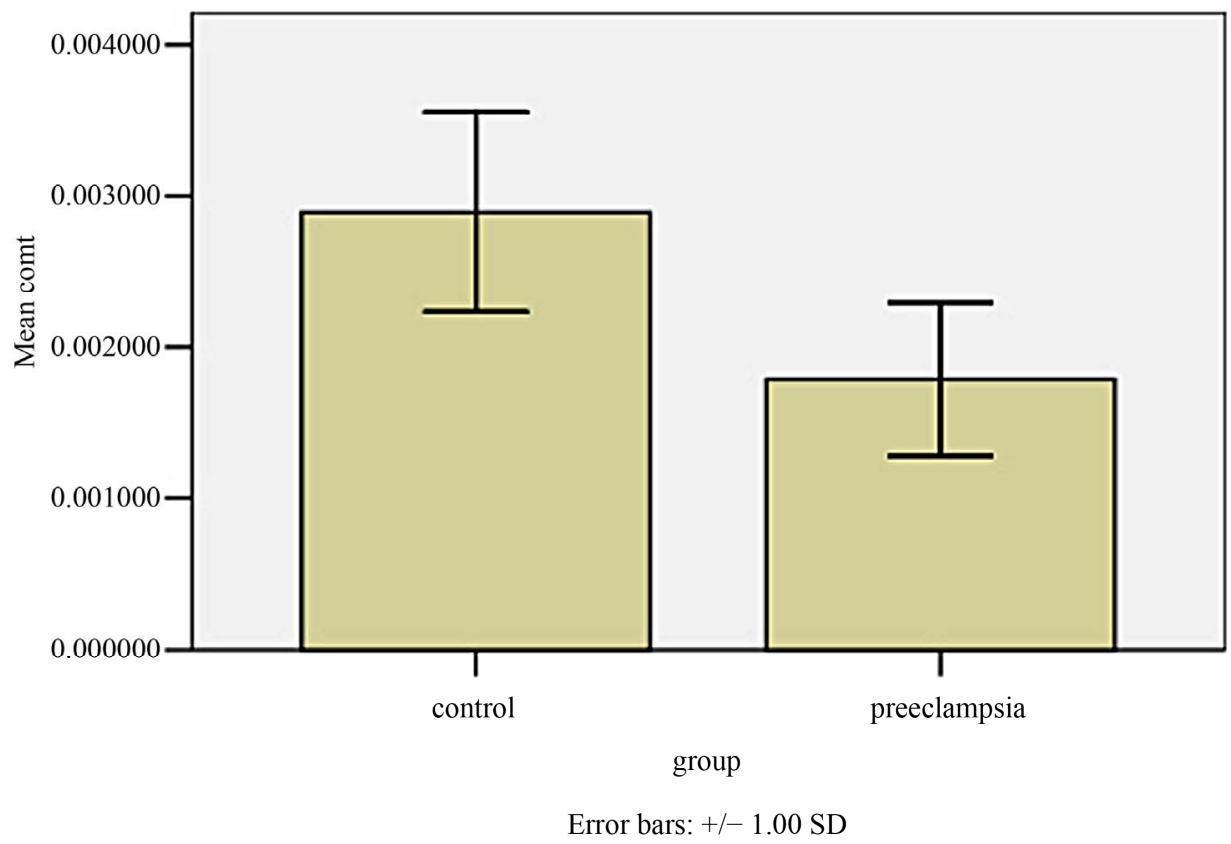

Figure 1. The COMT mRNA level in human placental tissues. The COMT mRNA level in preeclamptic group was much lower than those of normal control group $(t=5.145, P=0.000)$. 


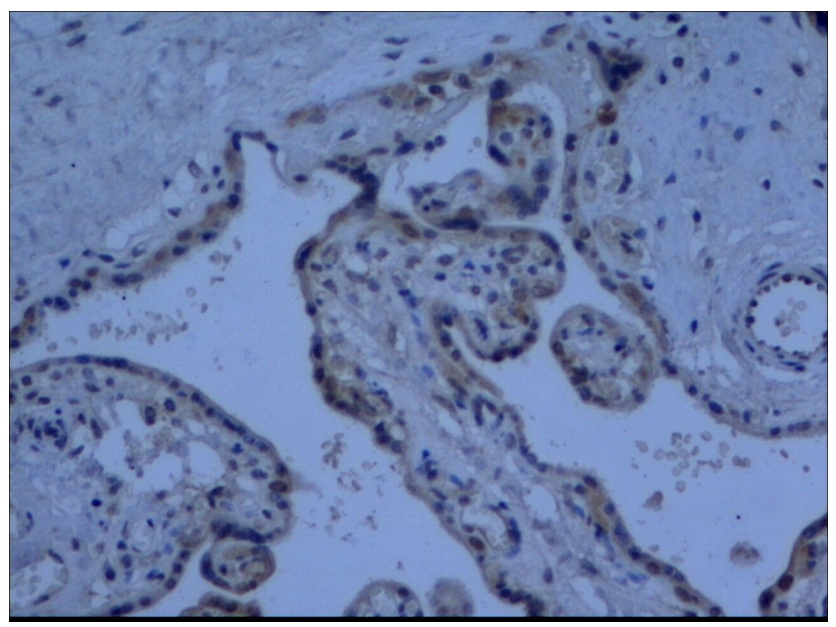

(a)

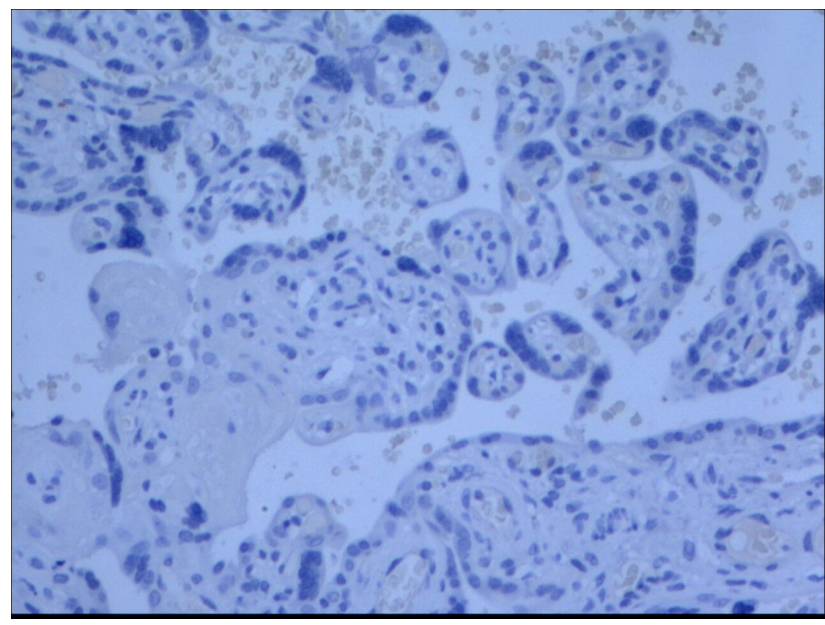

(c)

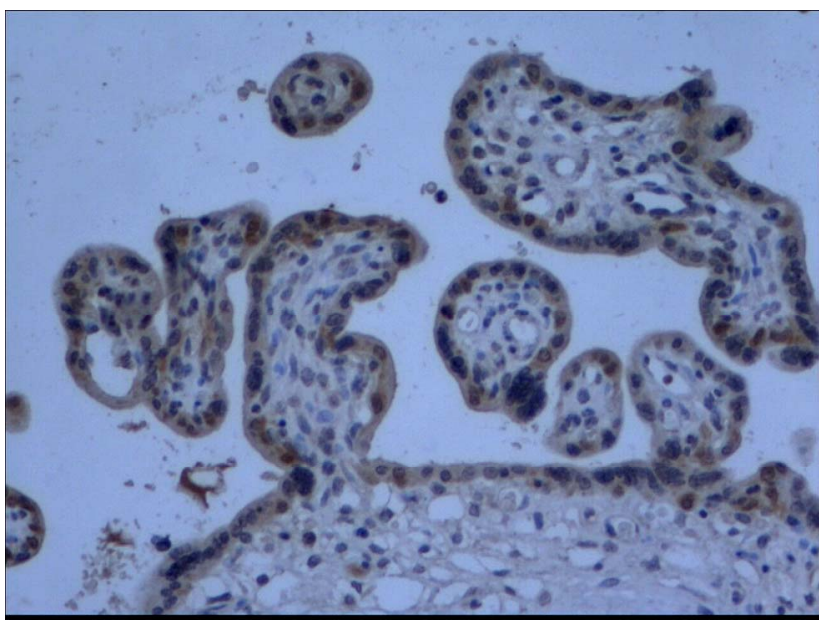

(b)

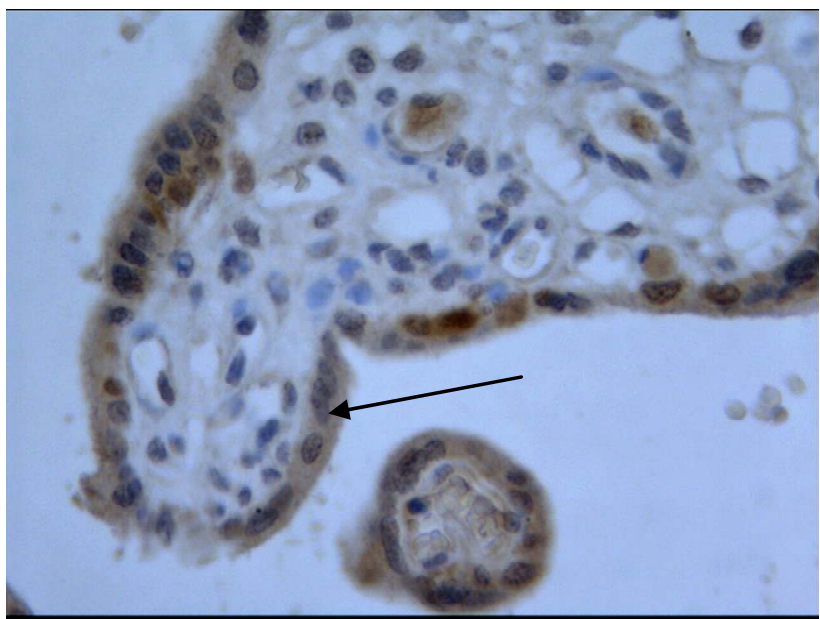

(d)

Figure 2. Immunohistochemistry images demonstrated COMT was localized in the layer of either syncytiotrophoblast or cytotrophoblasts (d). The lower expression of COMT was seen in preeclampsia (a) compared to that in normal pregnancy (b). No staining was evident in negative control sections $(\mathrm{c})(\times 200$ magnification).

Table 2. Correlations between clinical parameters and COMT mRNA Level.

\begin{tabular}{ccc}
\hline parameters & $\mathrm{r}$ & $\mathrm{P}$ \\
\hline MAP & -0.619 & 0.000 \\
Uric acid & -0.660 & 0.000 \\
24-hour urine protein & -0.717 & 0.000 \\
\hline
\end{tabular}

\section{DISCUSSION}

In this study we confirmed that reduced placental expression of COMT in preeclampsia compared with control term pregnancy at both the mRNA and protein levels. It was also demonstrated COMT-mRNA expression was negatively correlated with MAP, serum uric acid and 24hour urine protein.

COMT is a key enzyme involved in catecholamine and estrogen degradation [6]. It is active in both placenta and deciduas $[7,8]$. Decreased placental COMT activity was first reported to be associated with hypertension in pregnancy [7]. More recently, Kanasaki et al. demonstrated that pregnant mice deficient in COMT develop multiple functional and structural features of preeclampsia-like phenotype due to absence of 2-ME which is a metabolite of $17 \beta$-esteradiol generated by COMT [2]. Administration of 2-ME rescued the COMT-deficient mice from the preeclampsia-like syndrome without toxicity. The authors demonstrated that reduced placental COMT expression in the pathophysiology characteristics of preeclampsia. And this was confirmed by the pharmacologic study, in which the inhibition of COMT induced manifestations of preeclampsia in normal pregnant mice. And normal pregnant mice developed preeclampsia when given an inhibitor of a precursor of the 2-ME protein. But the clinical evidence about relationship between COMT and preeclampsia has been limited and inconsistent. Kanasaki examined placental COMT protein expression in only 6 preeclampsia women in the 


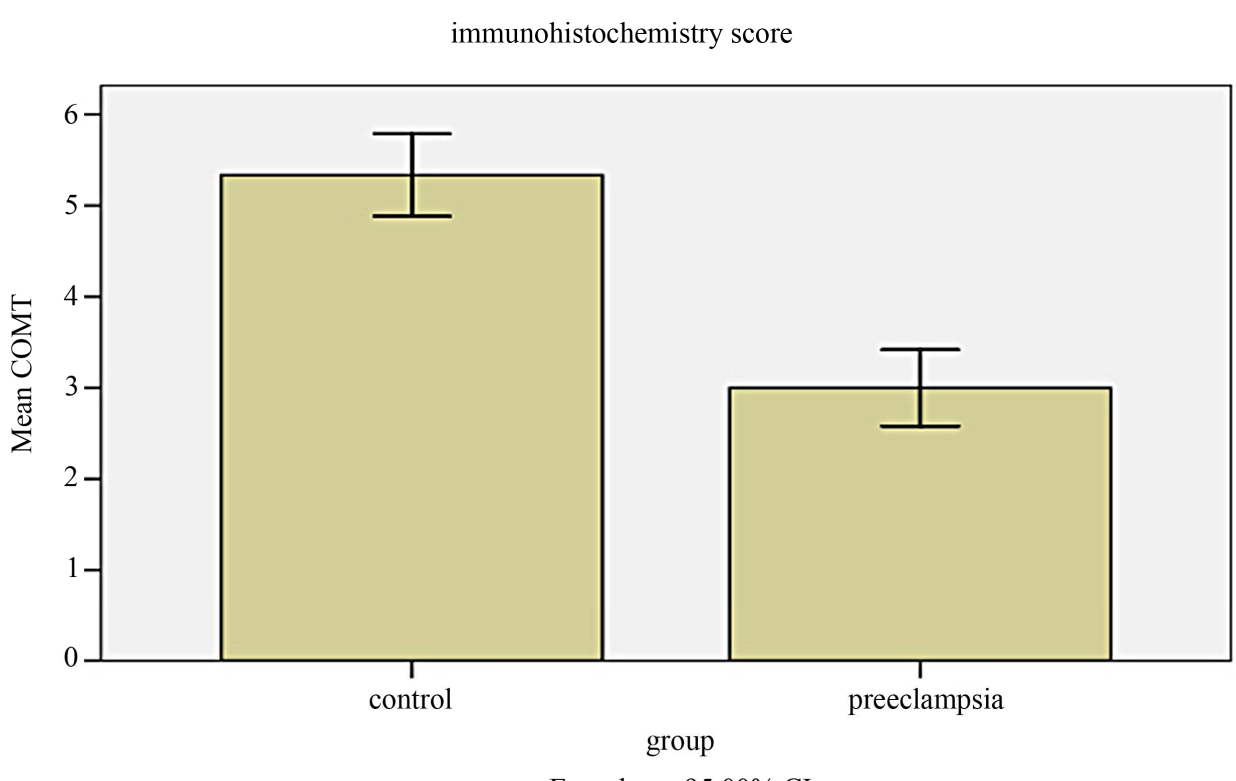

Error bars: $95.00 \%$ CI

Figure 3. Semiquantitative analysis indicated the expression of COMT significantly reduced in preeclamptic placentas compared to normal pregnancy $(P=0.01)$.

third trimester and Lee just 3 severe preeclampsia placentas $[2,3]$. Consistent with the results of Lee and Kanasaki, our study using placentas from term pregnancy at both the mRNA and protein levels confirmed an association between reduced COMT expression and preeclampsia. However, in the early onset severe preeclampsia group, Palmer reported that placental COMT expression was not different from that of term or preterm normotensive pregnant women at both the mRNA and protein levels [9]. Very recently, Seol also reported that placental COMT expression was not different between women with late onset severe preeclampsia and term normal pregnant women at protein level [10]. These discrepancies can be partially explained by the use of different study populations and detection methods. Furthermore, the studies had some limitations. The control group in Palmer's research included 14 normal-term pregnancies and 8 preterm pregnancies complicated by some diseases but not preeclampsia. The research compared the placental COMT mRNA level between the term pregnancies and the early-onset preeclampsia, and the gestational age was significantly different between the two groups (38.5 vs $31, P<0.001)$. The same limitation existed in Seol's study (gestational age: 38.1 vs $36.0, P=0.015$ ). There were no significant differences between the two groups with respect to gestational age in our study (38.45 vs 38.86, $P>0.05)$.

MAP, serum uric acid and urine protein were important indexes reflecting the severity of preeclampsia. This research firstly investigated the association between placental COMT expression and them in preeclampsia. We found placental COMT expression was significantly ne- gatively correlated with serum uric acid, urine protein and MAP respectively.

\section{CONCLUSION}

In conclusion, this study shows that the COMT mRNA and protein are reduced in placentas from term preeclampsia patients, and this is negatively correlated with MAP, uric acid and urine protein suggesting a role in the pathophysiological changes that occur in this disorder. Further longitudinal investigations regarding placental COMT polymorphism and 2-ME level are needed to explore the role of COMT in the pathophysiology of preeclampsia.

\section{REFERENCES}

[1] Hertig, A. and Liere, P. (2010) New markers in preeclampsia. Clinica Chimica Acta, 411, 1591-1595. doi:10.1016/j.cca.2010.07.020

[2] Kanasaki, K., Palmsten, K., Sugimoto, H., et al. (2008) Deficiency in catechol-O-methyltransferase and 2-methoxyoestradiol is associated with pre-eclampsia. Nature, 453, 1117-1121. doi:10.1038/nature06951

[3] Lee, S.B., Wong, A.P., Kanasaki, K., et al. (2010) Preeclampsia: 2-methoxyestradiol induces cytotrophoblast invasion and vascular development specifically under hypoxic conditions. American Journal of Pathology, 176, 710-720. doi:10.2353/ajpath.2010.090513

[4] Kenneth, L., Hall, D.R., Gebhardt, S. and Grove, D. (2010) Late onset preeclampsia is not an innocuous condition. Hypertens Pregnancy, 29, 262-270. doi:10.3109/10641950902777697

[5] Soslow, R.A., Dannenberg, A.J., Rush, D., et al. (2000) 
COX-2 is expressed in human pulmonary, colonic, and mammary tumors. Cancer, 89, 2637-2645.

doi:10.1002/1097-0142(20001215)89:12<2637::AID-CN CR17>3.0.CO;2-B

[6] Mannisto, P.T. and Kaakkola, S. (1999) Catechol-O-methyltransferase (COMT): Biochemistry, molecular biology, pharmacology, and clinical efficacy of the new selective COMT inhibitors. Pharmacological Reviews, 51, 593-628.

[7] Barnea, E.R., Maclusky, N.J., Decherney, A.H. and Naftolin, F. (1988) Catechol-o-methyl transferase activity in the human term placenta. American Journal of Perinatology, 5, 121-27. doi:10.1055/s-2007-999669

[8] Casey, M.L. and Macdonald, P.C. (1983) Characterization of catechol-O-methyltransferase activity in human ute- rine decidua vera tissue. American Journal of Obstetrics \& Gynecology, 145, 453-457.

[9] Palmer, K., Saglam, B., Whitehead, C., Stock, O., Lappas, M. and Tong, S. (2011) Severe early-onset preeclampsia is not associated with a change in placental catechol Omethyltransferase (COMT) expression. American Journal of Pathology, 178, 2484-2488. doi:10.1016/j.ajpath.2011.02.029

[10] Seol, H.J., Cho, G.J., Oh, M.J. and Kim, H.J. (2013) 2-Methoxyoestradiol levels and placental catechol-O-methyltransferase expression in patients with late-onset preeclampsia. Archives of Gynecology and Obstetrics, 287, 881-886. doi:10.1007/s00404-012-2663-1 